\title{
Co-relation between Sepsis Score and Blood Culture Report in Neonatal Septicaemia
}

\author{
$\mathrm{S} \mathrm{AFROZA}^{\mathrm{a}}$, F BEGUM ${ }^{\mathrm{b}}$
}

\section{Summary:}

Objective: To determine the clinical profile and to corelate the sepsis score with blood culture reports in neonatal septicaemia. Methods: Over a period of 6 months (1st week of June to 1st week of December'2005) 50 consecutive newborns with suspected septicaemia were enrolled for the study. It was a prospective study and septicaemia was suspected on the basis of clinical presentation like reluctance to feed, lethargy, fever, abdominal distension etc. Sepsis scoring was done for all of them immediately after enrollment into the study. Investigations like CBC, CRP and blood culture were sent for all the enrolled cases. Then the sepsis scores were compared with their blood culture reports to find out any correlation between them. The data analysis was done by SPSS software. Results: Among the 50 studied babies 31 were male and rest were female. Most of them were delivered by vaginal delivery (74\%) but no significant difference was observed among home and institutional delivery. During delivery 24 babies experienced some

\section{Introduction}

Neonatal sepsis is one of the major health problems throughout the world. Infections are a frequent and important cause of morbidity and mortality in newborn period'. As many as $2 \%$ of foetus are infected in utero and upto $10 \%$ of infants are infected in first month of life'. Data suggests that among four main causes of neonatal death, infection topped the list ${ }^{2}$. Every year an estimated 30 million newborns acquire infection and 1-2 million of these die ${ }^{3}$.

a. Prof. Syeda Afroza, (Appear in the article: S Afroza/Afroza S as applicable for the journal) MBBS, FCPS, Paed, DMEd (UK), MMEd (UK), Clinical Fellow of Neonatology (UK), FRCP (Edin), Professor (Paediatrics), Curriculum Development and Evaluation Centre for Medical Education, Mohakhali, Dhaka-1212.

b. Dr. Feroza Begum, MBBS, Asstt. Registrar, Department of Paediatrics, Institute of Child and Mother Health, Matuail Dhaka-1362

Address for correspondence: Prof. Syeda Afroza, (Appear in the article: S Afroza/Afroza $\mathrm{S}$ as applicable for the journal) MBBS, FCPS, Paed, DMEd (UK), MMEd (UK), Clinical Fellow of Neonatology (UK), FRCP (Edin), Professor (Paediatrics), Curriculum Development and Evaluation Centre for Medical Education, Mohakhali, Dhaka-1212.

Received: 9 July, 2006

Accepted: 7 March, 2007 problems of which $83.3 \%$ had perinatal asphyxia. About $59 \%$ of the studied babies were not exclusively breastfed. Majority of them (62\%) presented with reluctance to feed and $54 \%$ were preterm low birth weight. Fever and respiratory distress were present in 19 (38\%) and 18 (36\%) cases respectively. Forty two percent studied babies had positive sepsis score 5 and above. Regarding correlation of blood culture and sepsis score, 70\% culture positive cases had sepsis score 5 and above whereas 35\% of culture negative cases had the same score. Sensitivity and specificity of sepsis score was 70 and 65 respectively with CI interval 95\%. Conclusion: Sepsis score can be considered as an useful tool in the diagnosis of neonatal septicaemia specially where there is lack of investigational facilities. Before using this tool further evaluation is needed involving large sample size.

Key words: Neonatal septicaemia, sepsis score, blood culture.

(J Bangladesh Coll Phys Surg 2008; 26: 79-82)

Neonatal sepsis (also called septicaemia) is defined as a clinical syndrome characterized by signs of systemic infection and documented by a positive blood culture in the first four weeks of life ${ }^{1,4-7}$. Newborns of whole world specially those of third world countries are most vulnerable group for this illness. It is crucial to protect the newborns from infection as far as it is possible. In a poor country like ours, it is the responsibility of paediatricians (besides neonatologists) to address this serious neonatal health problems.

In developing countries the organisms causing neonatal sepsis and meningitis are different. A Bangladeshi study showed that there is considerable change in bacteriae causing neonatal sepsis and meningitis in 5 years period. $(1998-2003)^{8}$. In that study Klebsiella pneumoniae remained the leading pathogen comprising 23\% in 1998 and 23.4\% in 2003 in causing neonatal sepsis. S aureus was $17 \%$ and $6 \%$ respectively and Acinatobacter $6.7 \%$ and $20.4 \%$ respectively.

A number of prepartum and intrapartum obstetric complications are associated with increased 
incidence of neonatal sepsis. Examples are: premature onset of labour $(<37$ weeks of gestation $)^{7,9}$, premature or prolonged rupture of membrane $(>24 \text { hours })^{7,9,10}$ prolonged labour and excessive manipulation during labour ${ }^{9 \circ 10}$, intrapartum maternal fever ${ }^{7,9}$. Pre-term and low birth weight infants are at particular high risk of infection ${ }^{9}$. To diagnose a newborn with neonatal sepsis a careful maternal obstetric history regarding perinatal events should be taken to identify any risk factors. Sepsis score is an useful method for early and rapid diagnosis of neonatal sepsis. It can be considered as screening test for neonatal sepsis. This is specially useful in our context where there is limited facilities for investigations. This score was developed by Tollner U in $1982^{11}$. It has been recommended for easy application in our situation ${ }^{12}$.

\section{Methodology}

Type of the study: Descriptive study.

Place of the study: Neonatal care unit of ICMH.

Duration of the study: 6 months ( $1^{\text {st }}$ week of June to $1^{\text {st }}$ week of December'2005).

Sample size and sampling: Fifty (50) consecutive newborns by purposive sampling.

Inclusion criteria: suspected septicaemia on the basis of clinical presentation like reluctance to feed, lethargy, fever, abdominal distension.

Exclusion criteria: Perinatal asphyxia with features of HIE.

Sepsis score developed by Tollner U was used. Sepsis score data acquisition form includes 12 clinical and haematological parameters. Each component has some score e.g., skin colouration (Normal $=0$, moderate change $=2$, considerable change $=4$ ), microcirculation (Normal $=0$, impaired $=2$, considerably impaired $=3$ ), thrombocytopenia $(N o=0$, yes $=2$ ). Each component has been clearly explained in the form. The interpretation of the score: score $0-4.5=$ no sepsis; score $\geq 5$ observation range and suspicion of sepsis.

Sepsis score $\geq 5$ was considered as positive sepsis score in the present study. Study procedure: Sepsis scoring was done for all the studied babies immediately after enrollment into the study.
Investigations like $\mathrm{CBC}$ (Complete blood count), CRP(C-reactive protein) and blood culture were sent for all the enrolled cases. Then the sepsis scores were compared with their blood culture reports to find out any correlation between them.

The data analysis: was done by SPSS software.

\section{Results}

Among the 50 studied babies 31 were male and rest were female. Most of them were delivered by vaginal delivery $(74 \%)$ but no significant difference was observed among home and institutional delivery (Table I-III). During delivery 24 babies experienced some problems, of which $83.3 \%$ had perinatal asphyxia (Table IV). About $59 \%$ of the studied babies were not exclusively breastfed (Table V). Majority of them (62\%) presented with reluctance to feed and $54 \%$ were preterm low birth weight. Fever and respiratory distress were present in $19(38 \%)$ and $18(36 \%)$ cases respectively (Table VI). Blood culture was positive in $10(20 \%)$ and a quite good number of patients $21(42 \%)$ had positive sepsis score ie., 5 and above (Table VII). Regarding correlation of blood culture and sepsis score , 70\% culture positive cases had sepsis score 5 and above which was statistically significant. Among the culture negative cases only $35 \%$ had sepsis score 5 and above (Table VII). Sensitivity and specificity of sepsis score was 70 and 65 respectively with CI interval $95 \%$.

\section{Table I}

Sex distributions of studied babies $(N=50)$

\begin{tabular}{lcc} 
Sex & Number & Percent $(\%)$ \\
\hline Male & 31 & 62 \\
Female & 19 & 38 \\
\hline
\end{tabular}

Table II

Mode of delivery $(N=50)$

\begin{tabular}{lcc} 
Mode & Number & Percent $(\%)$ \\
\hline Vaginal & 37 & 74 \\
Caesarian & 13 & 26 \\
\hline
\end{tabular}

$\mathrm{P}$ value $=<.001$ 
Table III

Place of delivery $(N=50)$

\begin{tabular}{lcc} 
Place & Number & Percent $(\%)$ \\
\hline Home & 27 & 54 \\
Hospital & 23 & 46 \\
\hline P value $=<0.54$ & &
\end{tabular}

Table IV

\begin{tabular}{lcc}
\multicolumn{3}{c}{ Problem during delivery $(N=24)$} \\
Problems & Number & Percent $(\%)$ \\
\hline Perinatal asphyxia & 20 & 83.3 \\
Birth injury & 04 & 16.6 \\
\hline
\end{tabular}

Table V

Feeding pattern of studied babies $(N=49)$

\begin{tabular}{lcc} 
Feeding & No & Percent (\%) \\
\hline Exclusive breastfeeding & 20 & 40.8 \\
Artificial feeding & 3 & 6.1 \\
Mixed feeding & 6 & 12.2 \\
Breastfeeding after & 20 & 40.8 \\
pre-lacteal feeding & & \\
\hline
\end{tabular}

\section{Discussion}

Neonatal sepsis is one of the killer diseases of newborns. Specially when it occurs in the first week of life it can be a devastating neonatal problem. Male infants are more prone to develop infection ${ }^{5,9}$. In the present study $62 \%$ was male among the suspected neonatal sepsis babies. The resistance to infection in females is probably related to presence of mutant immunoregulatory genes located on the $\mathrm{X}$ chromosome ${ }^{13,14}$. Prolonged labour and excessive manipulation during labour may increase the incidence of neonatal sepsis 9,10 . Mode of delivery was pervaginal in $74 \%$ septic babies of present study and sepsis may be due to excessive manipulation during labour.

The frequent occurrence of foetal hypoxia and acidosis further impedes host defence mechanisms in small infants ${ }^{9}$ which may be true for the present study, where among 24 septic babies $83 \%$ had perinatal asphyxia.
If a previously healthy baby refuses to feed or reluctant to finish food, infection should be suspected. $7,12,15$, Reluctance to feed was the most common symptom in the present study, then was the prematurity and respiratory distress which may be the most common symptom of neonatal sepsis ${ }^{7,9}$.

Though neonatal sepsis (also called septicaemia) is characterized by signs of systemic infection and documented by a positive blood culture ${ }^{1,4-7}$ that is not true in $100 \%$ cases. Culture positivity may vary from less than $20 \%$ upto $70 \%$. In some developing countries blood culture was positive in $30.8 \%{ }^{16}$ and $42 \% 17$ respectively in otherwise proved neonatal sepsis which was $20 \%$ in the present study.

Sepsis score is an useful tool for early and rapid diagnosis of neonatal sepsis. It can be considered as screening test for neonatal sepsis. Recently a seven item weighted clinical score has been developed to diagnose late onset neonatal sepsis ${ }^{18}$. Culture positivity has been correlated with the sepsis score in the present study. Among the culture positive cases $70 \%$ had higher sepsis score i.e., 5 and above which was statistically significant. The sensitivity and specificity of sepsis score for the present study was 70 and 65 (with CI-95\%) respectively which is quite good and acceptable for our country.

\section{Conclusion}

It can be concluded from the present study that sepsis score can be used as a tool in the diagnosis of neonatal septicaemia specially where there is lack of investigational facilities. But it needs further evaluation involving large sample size.

\section{References}

1. Gotoff SP. Infections of the neonatal infant. In: Behrman RE, Kliegman RM, Jensson HB editors. Nelson Textbook of Paediatrics, $16^{\prime \prime}$ ed. Philladelphia: WB Sounders Company, 2002; p. 538-52.

2. Save the Children. State of World's Newborns. A Report from SNL, Save the Children (USA); 2003.

3. Stoll B. The global impact of neonatal infection. Clin Perinatol 1997; 24: 1-27

4. Gotoff SP and Behrman RE. Neonatal septicaemia. J Pediatr 1970; 76: 142-43.

5. Klin JO, Marcy SM. Bacterial sepsis and meningitis. In: Remington JS, Klein JO editors. Infectious diseases of foetuses and newborn infants. Philladelphia: WB Sounders Company 1983; p. 679-735. 
6. Hull D, Johnston DI editors. Essential Paediatrics. 2"d edition, Churchill Livingstone; 1990.

7. John P Clohery, Ann R Stark editors. Manual of Neonatal Care. 4'h edition. Lippincott Raven Publishers, Philadelphia; 1998.

8. Soman M, Green B, Daling J. Risk factors for early neonatal sepsis. Am J Epiodemiol 1985; 121: 712-19.

9. Pyati SP. Foetal and Neonatal Infections. In: Vidyasagar D editor. Textbook of Neonatology. Interprint, Mehta Offset Works, New Delhi 1987; p. 32-50.

10. Schlegel RJ, Bellante JA. Increased susceptibility of male to infection. Lancet 1969;2:826.

11. Tollner U. Early diagnosis of septicaemia in the newborn: clinical studies and sepsis score. Euro J Pediatr 1982; 138: 331-37.
12. Rashid MA. Neonatal Sepsis (An Update Review). Dissertation for FCPS Part II, 1988.

13. Purtillo DT, Sullivan JL. Immunological basis for superior survival of females. Am J Dis Child 1979; 128: 407.

14. Nyhan WL, Fousek KD. Septicaemia in the newborn. Pediatrics 1958; 22: 268-78. 15. Gandi GM, Roberton NRC editors. Lecture notes on Neonatology. Blackwell Scientific Publications Ltd. 1987.

16. Mokuolu AO, Jiya N, Adesiyun 00. Neonatal septicaemia in Illorin: bacterial pathogens and sensitivity pattern.. Afr J Med Sci 2002; 31: 127-30.

17. Kumher GD, Ramachandran VG, Gupta P. Bacterial analysis of blood culture isolates from neonates in a tertiary care hospital in India. J Health Popul Nutr. 2002; 20: 343-7.

18. Modi N, Carr R. Promising strategies for reducing burden of neonatal sepsis. Arch Dis Child (Fetal and Neonatal Ed) 2000; 83: F 150-53. 